Research Articles: Behavioral/Cognitive

\title{
Attention differentially affects acoustic and phonetic feature encoding in a multispeaker environment
}

https://doi.org/10.1523/JNEUROSCI.1455-20.2021

Cite as: J. Neurosci 2021; 10.1523/JNEUROSCI.1455-20.2021

Received: 8 June 2020

Revised: 28 September 2021

Accepted: 29 September 2021

This Early Release article has been peer-reviewed and accepted, but has not been through the composition and copyediting processes. The final version may differ slightly in style or formatting and will contain links to any extended data.

Alerts: Sign up at www.jneurosci.org/alerts to receive customized email alerts when the fully formatted version of this article is published.

Copyright (? 2021 Teoh et al.

This is an open-access article distributed under the terms of the Creative Commons Attribution 4.0 International license, which permits unrestricted use, distribution and reproduction in any medium provided that the original work is properly attributed. 


\title{
Attention differentially affects acoustic and phonetic feature
} encoding in a multispeaker environment

\author{
Emily S. Teoh ${ }^{1}$, Farhin Ahmed ${ }^{2}$, Edmund C. Lalor ${ }^{1,2}$ \\ ${ }^{1}$ School of Engineering, Trinity Centre for Biomedical Engineering, and Trinity College Institute of Neuroscience, \\ Trinity College, University of Dublin, Dublin 2, Ireland \\ ${ }^{2}$ Department of Neuroscience, Department of Biomedical Engineering, and Del Monte Neuroscience Institute, \\ University of Rochester, Rochester 14627, NY, USA
}

\section{Corresponding author email address: Edmund_Lalor@urmc.rochester.edu}

Running title: Attention enhances phonetic feature encoding

\section{Abstract}

Humans have the remarkable ability to selectively focus on a single talker in the midst of other competing talkers. The neural mechanisms that underlie this phenomenon remain incompletely understood. In particular, there has been longstanding debate over whether attention operates at an early or late stage in the speech processing hierarchy. One way to better understand this is to examine how attention might differentially affect neurophysiological indices of hierarchical acoustic and linguistic speech representations. In this study, we do this by using encoding models to identify neural correlates of speech processing at various levels of representation. Specifically, we recorded EEG from fourteen human subjects (nine female and five male) during a "cocktail party" attention experiment. Model comparisons based on these data revealed phonetic feature processing for attended, but not unattended speech. Furthermore, we show that attention specifically enhances isolated indices of phonetic feature processing, but that such attention effects are not apparent for isolated measures of acoustic processing. These results provide new insights into the effects of attention on different pre-lexical representations of speech, insights that complement recent anatomical accounts of the hierarchical encoding of attended speech. Furthermore, our findings support the notion that - for attended speech - phonetic features are processed as a distinct stage, separate from the processing of the speech acoustics.

\section{Significance Statement}

Humans are very good at paying attention to one speaker in an environment with multiple speakers. However, the details of how attended and unattended speech are processed differently by the brain is not completely clear. Here we explore how attention affects the processing of the acoustic sounds of speech as well as the mapping of those sounds onto categorical phonetic features. We find evidence of categorical phonetic feature processing for attended, but not unattended speech. Furthermore, we find evidence that categorical phonetic feature processing is enhanced by attention, but not for acoustic 
processing. These findings add an important new layer in our understanding of how the human brain solves the cocktail party problem.

\section{Introduction}

The ability to focus on a single talker amidst multiple sounds is essential for human communication. Cherry (1953) brought this phenomenon to the fore, investigating our behavioral capacity for selective auditory attention. His study stimulated subsequent inquiries and the proposal of various theories of attention (Broadbent 1958; Moray 1959; Deutsch and Deutsch 1963; Treisman 1964; Johnston and Wilson 1980). These theories model attention as a selective filter that rejects the unattended message beyond a certain information processing stage. However, whether this filter operates at an early or late stage of speech processing is still unresolved.

In recent years, neuroscience has sought to contribute to this debate by leveraging our increased understanding of the hierarchical nature of speech processing in the human cortex. In particular, to construe meaning from sound, the cortex is posited to compute several intermediate levels of increasingly abstract representations in different functionally specialized regions of a hierarchically organized cortical network (Hickok and Poeppel 2007; Peelle et al. 2010; Rauschecker and Scott 2009; DeWitt and Rauschecker 2012; Kell et al. 2018). For example, single talker neuroimaging studies have revealed that core auditory regions code low-level features which are combined in higher areas to yield more abstract neural codes (Binder et al. 2000; Davis and Johnsrude 2003; Okada et al. 2010). And in terms of multi-talker selective attention, studies have shown that primary auditory cortical responses represent all talkers irrespective of attentional state (J. O'Sullivan et al. 2019), but in "higher" areas such as the superior temporal gyrus (STG), only the attended speaker is represented (Mesgarani and Chang 2012; Zion Golumbic et al. 2013; J. O’Sullivan et al. 2019). Additionally, EEG/MEG studies examining the latency of neural responses (which can be considered a rough proxy measure of processing at different hierarchical stages) have shown that all talkers are co-represented in early components, with distinct responses to the attended speaker appearing only in later components (Ding and Simon 2012b; Power et al. 2012; Puvvada and Simon 2017). Importantly, this specificity is not something that is necessarily true for more simplistic non-speech stimuli and tasks (e.g., Power et al., 2011). 
As well as understanding how attention affects processing in different brain areas and at different latencies, we also wish to understand how attention influences the encoding of the different speech representations that are thought to be computed by this hierarchical network. In recent years, encoding/decoding methods (Crosse et al. 2016; Holdgraf et al. 2017) have revealed neural indices of a number of these representations - from low-level acoustics to semantics - in brain responses to continuous natural speech (Ahissar et al. 2001; Brodbeck et al., 2018; Broderick et al. 2018; Daube et al., 2019; Di Liberto et al. 2019; Di Liberto et al., 2015; Mesgarani et al. 2014; Tang et al., 2017; Teoh et al., 2019). And it has been shown that neural indices of lexical (Brodbeck et al., 2018) and semantic (Broderick et al. 2018) processing can only be found for attended speech, in contrast to acoustically-driven measures like those based on the amplitude envelope that are less affected by attention (Brodbeck et al., 2018). Taken together, the results from these anatomical, time-resolved, and representational approaches support the notion that higher-order regions (and higher-level representations) are more greatly modulated by attention.

In this study, we explore how attention modulates neural indices of speech processing at prelexical levels. Specifically, we aim to answer two main questions. Our first question is: does the brain process speech at the level of phonemes and, if so, does it do so for both attended and unattended speech? The notion of pre-lexical processing at the level of phonemes has received neuroscientific support in recent years (Di Liberto et al., 2015; Khalighinejad et al., 2017; Brodbeck et al., 2018; Gwilliams et al., 2020; Mesgarani et al. 2014; Chang et al. 2010) . But the idea has also been challenged, with some recent research suggesting that what appear to be categorical responses to phonetic features might actually be responses to simple acoustic features (Daube et al., 2019). Here, to address this debate, we explore brain responses to speakers with very different acoustics (i.e., male and female). The rationale here is that a greater increase in acoustic variability across the same phonemes will improve our ability to distinguish categorical brain responses to different phonetic features from brain responses driven by the speech acoustics. Our second question is: at what hierarchical levels does attention influence processing? We hypothesize that we will see stronger attention effects on isolated measures of phonetic feature processing than on isolated measures of acoustic processing. This would support the idea of hierarchical attention effects suggested by previous studies by showing a dissociation in the influence of attention on different aspects of sublexical processing. 


\section{Methods}

$101 \quad \underline{\text { Subjects }}$

102

103

Fourteen subjects (nine female and five male) between the ages of 19 and 30 participated in the experiment. All subjects were right-handed and spoke English as their primary language. Subjects reported no history of hearing impairment or neurological disorder. Each subject provided written informed consent prior to testing and received monetary reimbursement. The study was approved by the Research Subjects Review Board at the University of Rochester. Some of the data used here (the first 10 trials) were analyzed differently for a previous study (Teoh and Lalor 2019).

\section{$\underline{\text { Stimuli and Procedures }}$}

Subjects undertook 40 one-minute trials in two separate blocks. Stimuli consisted of two works of fiction, one narrated by a female talker and the other by a male talker. The stimuli were taken from two Sherlock Holmes novels: "A Study in Scarlet" (narrated by a female speaker) \& "The Hound of the Baskervilles" (narrated by a male speaker). The stories were narrated by British speakers. Silent gaps in the audio exceeding $0.3 \mathrm{~s}$ were truncated to $0.3 \mathrm{~s}$ in duration.

The stimuli were filtered using Head-Related Transfer Functions (HRTFs), giving rise to the perception that the talkers were at 90 degrees to the left and right of the subject. The HRTFs used were obtained from the CIPIC database (Algazi et al., 2001). Subjects were always instructed to attend to one of the two talkers - a counterbalanced paradigm was employed in which, over the course of the experiment, they would have attended to both male and female talkers and at both locations. In terms of content, the attended story segments were presented contiguously (i.e., each trial beginning where previous one ended), but the unattended story segments were presented in a random order. Each trial lasted exactly $60 \mathrm{~s}$, with no special effort made to end the trial at a natural break in the story.

Before the experiment, subjects were asked to minimize motor activities and to maintain visual fixation on a crosshair centered on the screen during trials. After each trial, subjects were required to answer four multiple-choice comprehension questions on each of the stories (attended and unattended). 
128 All stimuli were sampled with a frequency of $44100 \mathrm{~Hz}$ and were presented using Sennheiser 129 HD650 headphones and Presentation software from Neurobehavioral Systems 130 (http://www.neurobs.com).

\section{Data Acquisition and Pre-processing}

The experiment was conducted in a soundproof room. A Biosemi ActiveTwo system was used to record EEG data from 128 electrode positions on the scalp as well as two electrodes over the mastoid processes (all digitized at $512 \mathrm{~Hz}$ ).

EEG data were re-referenced to the mastoids. Automatic bad channel rejection and interpolation was performed. A particular channel was deemed as bad if the standard deviation of the channel was lower than a third of or exceeded three times the mean of the standard deviation of all channels. In place of the bad channel, data were interpolated from the four nearest neighboring electrodes using spherical spline interpolation (Delorme and Makeig 2004). To decrease subsequent processing time, data were downsampled to $128 \mathrm{~Hz}$. Data were filtered between $0.2-$ $8 \mathrm{~Hz}$ using a zero-phase shift FIR filter with Kaiser window (filter order was 20000 for the 0.2 $\mathrm{Hz}$ high pass filter and 1000 for the $8 \mathrm{~Hz}$ low pass filter; maximum stopband attenuation was -60 $\mathrm{dB}$ ). Low delta-band frequencies (down to $0.2 \mathrm{~Hz}$ ) were included as they have previously been found to be important for speech processing (Teoh et al, 2019).

\section{Speech Representations}

We computed acoustic and phonetic speech representations of both the attended and unattended stories (Figure 1A depicts these representations for an excerpt taken from one of the audio clips).

Acoustic:

- Spectrogram (s): The GBFB toolbox (Schädler and Kollmeier 2015) was used to extract the spectral decomposition of the time-varying stimulus energy in 31 mel-spaced bands with logarithmic compressive nonlinearity. The spectrogram representation computed in this way is consistent with (Daube et al., 2019), where it was shown to better predict neural activity than other techniques.

- Spectrogram Derivative $(s D)$ : The temporal derivative of each spectrogram channel was computed, and then half-wave rectified. This represents an approximation of the onsets in 
the acoustics and was shown to contribute to predicting neural responses in previous research (Daube et al., 2019).

Phonetic:

- Phonetic features $(f)$ : Phoneme-level segmentation of the stimuli was first computed using FAVE-Extract (Rosenfelder et al. 2014) and the Montreal Forced Aligner (McAuliffe et al. 2017) via the DARLA web interface (Reddy and Stanford 2015). The phoneme representation was then mapped into a space of 19 features based on the mapping described previously (Chomsky and Halle 1968; Mesgarani et al. 2014). The features describe the articulatory and acoustic properties of the phonetic content of speech (e.g., bilabial, plosive, voiced, obstruent). Based on the onset time of each feature, a multivariate time-series binary matrix (19 features by samples) was produced.

- Phonetic feature onsets $(f o)$ : Univariate vector of the onsets of all phonetic features (i.e., a summary measure of all onsets in $f$ without categorical discrimination). This was included as an additional control. Specifically, if the $f$ representation above fails to improve EEG prediction beyond the fo representation, it would suggest that the encoding of individual phonetic features is not reflected in EEG data.

\section{Model fitting: Multivariate Linear Regression}

The acoustic and phonetic representations for both attended and unattended speech described above were normalized and mapped to the concurrently recorded 128-channel EEG signals. Multivariate regularized linear regression was employed to relate the features to the recorded EEG data, where each EEG channel is estimated to be a linear transformation of the speech features over a range of time lags. This transformation is described by the temporal response function (TRF). For a particular speech feature, this operation can be represented mathematically as following (Crosse et al. 2016):

$$
r(t, n)=\sum_{\tau} w(\tau, n) s(t-\tau)+\epsilon(t, n)
$$


where $r(t, n)$ is the neural (EEG) response at channel, $n$ and time point, $t=1 \ldots T, s(t-\tau)$ is the multivariate stimulus representation at a lag, $\tau, w(\tau, n)$ is the transformation (TRF) of the stimulus at lag $\tau$, and $\epsilon(t, n)$ is the residual response not explained by the model.

The TRF is estimated by minimizing the mean square error between the actual neural response, $r(t, n)$, and the response predicted by the transformation, $\hat{r}(t, n)$. In practice, this can be solved by using reverse correlation. We use the mTRF toolbox (Crosse et al. 2016https://sourceforge.net/projects/aespa/), which solves for the TRF (w) using reverse correlation with ridge regression:

$$
w=\left(S^{T} S+\lambda I\right)^{-1} S^{T} r
$$

where $\lambda$ is the ridge regression parameter, $I$ is the identity matrix, and the matrix $S$ is the lagged time series of the stimulus matrix, $s$. The TRF approach can be used to relate multiple features of the stimuli to the ongoing EEG simultaneously by extending the lagged stimulus matrix to include the various features (more details can be found in Crosse et al. 2016). The ridge regression parameter was tuned using leave-one-out cross validation. That is, for each subject, we trained a separate TRF on each of $n-1$ trials $(\mathrm{n}=40)$ for a wide range of $\lambda$ values $\left(1 \times 10^{-1}\right.$, $1 \times 10^{0}, \ldots, 1 \times 10^{6}$ for the model comparison analysis, and $2^{-2}, 2^{-1}, \ldots, 2^{37}$ for the partial correlation analysis), computed the average TRF across trials for each $\lambda$, then tested the TRFs on the $n$th trial. This was repeated $n$ times, rotating the trial to be tested each time. For each subject, the single $\lambda$ value that maximized the Pearson's correlation coefficient between the actual and predicted neural response averaged over all trials and over all channels was selected. It is worth noting here that the optimal $\lambda$ parameter value did vary somewhat across subjects. This was not surprising, considering the fact that strength of EEG responses to speech (and hence their signalto-noise ratio) will vary across subjects due to differences in their cortical fold configuration and skull thickness, etc. However, the optimal $\lambda$ parameter typically fell into the intermediate region of our tested range, far from the lowest value. This indicates that incorporating regularization improved the model prediction accuracies.

The TRF mapping from stimulus to EEG was computed over a range of time lags, reflecting the idea that changes in the features of the ongoing stimulus are likely to produce effects in the ongoing EEG in that interval. Specifically, we chose the interval 0 to $300 \mathrm{~ms}$ based on previous 
210 EEG-based speech studies, where no visible response was present outside this range when 211 considering EEG responses to acoustic and phonetic features (Di Liberto et al., 2015; Lalor and 212 Foxe 2010). We quantified how well each speech representation related to the neural data using leave-one-trial-out cross-validation (as described above), with Pearson's correlation coefficient as our metric of prediction accuracy. Because the cross-validation procedure takes the average of the validation metric across trials, the models are not biased toward the test data used for crossvalidation (Crosse et al. 2016).

To evaluate whether a feature contributed independently of all other features in predicting the neural data, we also computed the partial correlation coefficients (Pearson's $r$ ) between the EEG predicted by each measure's model with the actual recorded EEG after controlling for the effects of all other features. Specifically, we fit separate cross-validated forward models/TRFs on each of the four speech representations $(s, s D, f$ and $f o$ ) and predicted EEG based on those models. Then, for the three features to be partialled out (e.g., $s, s D$, and $f o$ ), we used cross-validation to optimally predict our EEG data. We then concatenated these three EEG predictions into one matrix, Z. And, finally, we used the MATLAB built-in function partialcorr $(X, Y, Z)$ where $X=$ the actual recorded EEG, $\mathrm{Y}=$ the predicted EEG in response to the feature of interest (the feature whose unique contribution is to be identified, e.g., $f$ ), and $\mathrm{Z}=$ the concatenated predicted EEGs in response to the other features (features that are to be partialled out). This function computes the partial correlation coefficients between $\mathrm{X}$ and $\mathrm{Y}$, while controlling for the variables in $\mathrm{Z}$ (Fisher 1924).

Incidentally, we sought to confirm the validity of our partial correlation approach using a second approach. In this approach, we fit one model to three predictors simultaneously using crossvalidation (e.g., the $s, s D$ and $f o$ features). Then we used that model to predict the EEG data and subtracted that predicted data from the real data to leave us with residual EEG. Finally, (again using cross validation), we predicted this residual EEG using the left out predictor (e.g., $f$ ), to see if this predictor had any power to predict unique variance in the original EEG. This analysis produced the same pattern of result as the approach described in the previous paragraph.

\section{$\underline{\text { Statistical Testing }}$}

To test that a partial correlation coefficient is above chance level, we performed non-parametric permutation testing. The predicted EEG activity for each model's representation was permuted 
240 across trials such that they were matched to the actual EEG of a different trial, and partial 241 correlation coefficients were computed, controlling for the effects of all other features. This was 242 done 1000 times for each subject to establish a distribution of chance-level prediction accuracies. 243 To perform group-level statistical testing, we generated a null distribution of group means: one

[Fig 1 about here]

Figure 1: (A) Speech representations: The first row depicts an acoustic waveform of an excerpt taken from one of the stimuli. Subsequent rows show the computed acoustic and phonetic representations for that excerpt. (B) Analysis framework: Cross-validation is used to train forward models mapping the different attended and unattended speech representations to EEG. These models are then used to predict left-out EEG. Pearson's correlation is used to evaluate model accuracy, and the prediction accuracies of acoustic and phonetic models are compared. 


\section{Results}

Subjects were found to be compliant in carrying out the behavioral task (Figure 2A). The average questionnaire accuracy was $73.2 \pm 3.2 \%$ when subjects were tested on the attended story and $27.2 \pm 1.6 \%$ for unattended stimuli (theoretical chance level is $25 \%$ as there are four possible answers to each question).

As mentioned above, our study had two specific questions: 1) does the brain process speech at the level of phonemes and, if so, does it do so for both attended and unattended speech? And, 2) at what hierarchical levels does attention influence processing? To address both questions, we chose a two-stage data analysis strategy. The first stage sought to answer the first question. It involved comparing the performance of different models in predicting EEG responses to speech. More specifically, it involved assessing whether or not the inclusion of a categorical phonetic feature representation of speech $(f)$ could improve the prediction of EEG beyond that obtained using several other acoustic $(s, s D)$ and phonetic $\left(f_{o}\right)$ features. Furthermore, we aimed to assess whether any such improved prediction would hold for both attended and unattended speech. The second analysis was aimed at answering the second question. This strategy involved identifying the ability of each stimulus feature to predict unique variance in the EEG responses (while controlling, or partialling out, the predictive contributions of the other features), and then assessing whether that unique predictive contribution was affected by attention. While these two data analyses are closely related, each is targeted at one of our specific complementary questions.

\section{Model comparisons reveal that responses to attended talkers reflect phonetic-level processing}

We assessed the performance of attended and unattended models that were trained to predict EEG responses using the acoustic and phonetic feature representations extracted from the speech stimulus (Figure 2B). The Pearson's correlation between predicted and actual EEG activity, averaged across the same 12 bilateral fronto-temporal channels used in Di Liberto et al., (2015) and Daube et al. (2019), was used as our metric of prediction accuracy for each model (although the same pattern of results was observed if all 128 channels were used). All individual features could predict EEG above chance on a group level (non-parametric permutation test, $\mathrm{p}=0.999 \mathrm{e}-3$ ). Now, the feature spaces used here are not independent, so the individual model predictions are not a pure representation of the extent to which EEG tracks these features. The phonetic features 
representation $(f)$ overlaps with the spectrogram representation $(s)$ in that if every phoneme is always spoken the same way, then the two representations would be equivalent. The phoneme onset $(f o)$ representation marks the same time-points as the phonetic feature representation $(f)$, except that it does not contain feature category information. The spectrogram derivative $(s D)$, an approximation of acoustic onsets, contains peaks that overlap with those of the phoneme onset ( $f o$ ) representation. Thus, the processing of a particular feature was assessed by examining whether adding that feature improved prediction of the EEG responses beyond a model built from other features (Figure 2C). We were particularly interested in establishing whether the three other features could improve prediction accuracy beyond using spectrograms alone, and if phonetic features contributed unique predictive power beyond all other features. Importantly for comparing the present study with previous studies using this approach (Di Liberto et al., 2015; Daube et al., 2019), our experiment involved speakers with markedly different acoustics (i.e., a male speaker and a female speaker). This means an increase in the variability of the acoustic representation $(s)$ for the same phonetic features. As such, the phonetic feature representation should only improve predictions if there are consistent responses to the same phonetic features despite their acoustic variation. In order to test this idea, we performed pairwise statistical testing of the joint model prediction accuracies - these results are shown in Table 1.

[Fig 2 about here]

Figure $2 \mid$ (A) Behavioral (comprehension questionnaire) results - dots indicate individual subject performance. Theoretical chance level is $25 \%$ (multiple-choice test with four options) and is indicated by the dashed line (B) Prediction accuracies of individual acoustic and phonetic feature spaces (as labelled on horizontal axes) for the attended and unattended stimuli. There is redundancy between these features, so joint modelling was performed to gauge in particular whether phonetic features adds unique predictive power beyond other features (C) Prediction accuracies of joint feature spaces for attended and unattended stimuli $(* * \mathrm{p}<0.01$, two-tailed Wilcoxon sign-ranked test, FDR corrected). 
Table 1 | Joint model comparison statistics: Wilcoxon two-sided signed rank test results for attended and unattended stimuli (columns) along with the Bayes factors $\left(\mathrm{BF}_{10}\right)$. Each set of rows tests a different statistical question, as specified by the baseline. Terms being evaluated are to the left of the '>' symbol. Bolded values indicate significant improvement over baseline.

Attended

Unattended

\begin{tabular}{|c|c|c|}
\hline \multicolumn{3}{|l|}{ baseline: $s$} \\
\hline \multirow[t]{2}{*}{$s+f>$ baseline } & $\mathrm{p}=\mathbf{0 . 0 0 1 2}, \mathrm{z}=\mathbf{3 . 2 3 3 0}$ & $\mathrm{p}=0.4326, \mathrm{z}=0.7847$ \\
\hline & $B F_{10}=8.0159$ & $\mathrm{BF}_{10}=0.2790$ \\
\hline \multirow[t]{2}{*}{$s+s D>$ baseline } & $p=0.0023, z=3.0447$ & $p=0.0023, z=3.0047$, \\
\hline & $\mathrm{BF}_{10}=63.9041$ & $B F_{10}=13.2765$ \\
\hline \multirow[t]{2}{*}{$s+f_{O}>$ baseline } & $p=0.0015, z=3.1708$ & $\mathrm{p}=0.7299, \mathrm{z}=0.3453$ \\
\hline & $\mathrm{BF}_{10}=2.1335$ & $\mathrm{BF}_{10}=0.3035$ \\
\hline \multicolumn{3}{|l|}{ baseline: $s+s D$} \\
\hline \multirow[t]{2}{*}{$s+s D+f>$ baseline } & $p=0.0043, z=2.8563$ & $\mathrm{p}=0.1981, \mathrm{z}=1.2869$ \\
\hline & $\mathrm{BF}_{10}=7.1414$ & $\mathrm{BF}_{10}=0.6082$ \\
\hline \multicolumn{3}{|l|}{ baseline: $s+f o$} \\
\hline \multirow[t]{2}{*}{$s+f o+f>$ baseline } & $p=0.0023, z=3.0447$ & $\mathrm{p}=0.6378, \mathrm{z}=0.4708$ \\
\hline & $\mathrm{BF}_{10}=\mathbf{5 9 . 7 0 5 9}$ & $\mathrm{BF}_{10}=0.2829$ \\
\hline \multicolumn{3}{|l|}{ baseline: $s+f o+s D$} \\
\hline \multirow[t]{2}{*}{$s+f_{o}+s D+f>$ baseline } & $p=0.0076, z=2.6680$ & $\mathrm{p}=0.4703, \mathrm{z}=0.7219$ \\
\hline & $\mathrm{BF}_{10}=13.9134$ & $\mathrm{BF}_{10}=0.4912$ \\
\hline
\end{tabular}

In general, we found that adding model features tended to improve EEG prediction performance for attended speech, with fewer improvements visible for unattended speech. Of particular relevance to our original question, we found that including phonetic features $(f)$ significantly improved prediction over the acoustic $(s, s D)$ features when subjects were attending to the speech $(p=0.0043)$; but this improvement was not observed for the unattended speech ( $p=$ 0.1981). Importantly, the addition of phonetic features also improved prediction when compared with a combination of acoustic features and phoneme onsets $\left(f_{o}\right)$ in the attended case $(\mathrm{p}=$ 
337 0.0076), indicating that the inclusion of specific phonetic feature categories carries unique and 338 important information. The acoustic onset (i.e., spectrogram derivative) representation displayed 339 a different pattern of results - including this measure along with the spectrogram (i.e., $s+s D$ ) improved prediction accuracy over spectrogram alone for both attended and unattended stimuli, suggesting that it is less strongly modulated by attention. For unattended speech, it is also worth noting that, while the phonetic feature representation did not improve the prediction of EEG responses over that based on the acoustic $(s+s D)$ or combined acoustic and phoneme onset $(s+s D+f o)$ features, the absence of evidence is not evidence of absence. Indeed, the Bayes factor scores for these two comparisons were $\mathrm{BF}_{10}=0.6082$ and $\mathrm{BF}_{10}=0.4912$, respectively. Both of these are larger than 0.33 , indicating we do not have strong evidence in support of the null hypothesis. As such, while we saw no evidence for phonetic feature processing of unattended speech, we cannot claim that it definitely is not happening. However, the evidence in support of such processing for attended speech is strong.

While our results provide evidence for phoneme level processing of attended speech, but no such evidence for unattended speech, the above analysis does not allow us to answer the second question our study set out to examine. In particular, the above results do not allow us to test the hypothesis that attention effects are stronger on measures of phonetic feature processing than on measures of acoustic processing. One might be tempted to conclude from the results in Fig 2B that attention is affecting processing at the level of acoustics (compare the prediction accuracies of the $s$ model for attended [left panel] vs unattended [right panel] speech). However, as discussed above, the $s$ and $f$ representations are likely to be highly correlated (given that the acoustics for a given phoneme will tend to be correlated across utterances of that phoneme). As such, if one were to observe an attention effect on the $s$ model alone, one would not be able to conclude that attention is affecting low-level acoustic processing. Rather, it could be operating at the level of phonemes and simply appearing in the $s$ model performance via correlation. Therefore, in order to answer our study's second question, we need another approach.

Variance partitioning analysis reveals that attention enhances isolated measures of phonetic feature processing, but not acoustic processing

Given the aforementioned redundancies between the predictions of feature spaces, we were also 366 interested in more clearly isolating the unique contribution of each feature. To do so, we 
employed a partial correlation approach to control for the predictions of all other representations. The unique predictive power of each model is shown in Figure 3A (shown here for an average across 12 channels, although including all 128 channels revealed the same pattern of results). On a group level, all features made unique, significant contributions to the EEG predictions except the spectrogram derivative models (attended and unattended), and the unattended phonetic features model (non-parametric permutation test; $s D$ attended: $\mathrm{p}=0.0619 ; s D$ unattended: $\mathrm{p}=0.1788 ; f$ unattended: $\mathrm{p}=0.1788$; all other models: $\mathrm{p}<0.05$ ). When comparing attended and unattended models, we found a significant effect of attention for phonetic features (two-tailed Wilcoxon signed rank test; $\mathrm{p}<0.05$, FDR corrected, $\mathrm{BF}_{10}=12.56$ ), but not for any of the other representations (two-tailed Wilcoxon signed rank test; FDR corrected; $s: \mathrm{p}=0.2412, \mathrm{BF}_{10}=$ 0.54; $s D: \mathrm{p}=0.3910, \mathrm{BF}_{10}=0.41 ; f o: \mathrm{p}=0.1726, \mathrm{BF}_{10}=0.59$ ). Again, the Bayes factor scores for these three latter results are all less than 1 , but greater than 0.33 . As such, we cannot definitively claim that there is no effect of attention on the $s, s D$, or $f o$ representations. However, our data do not provide any evidence of such an effect, unlike the strong effect we see for phonetic features. This strong effect for phonetic features, but not the other representations, is clearly visible in when visualizing the topographic distributions for the unique predictive power of each model (Figure 3B).

We think the above approach of explicitly testing the unique predictive power of different speech representations is a good way to explore the hierarchical processing of natural speech. However, further insight could potentially be gleaned by investigating how the EEG is affected by attention at different temporal delays relative to the speech stimulus. Indeed, in previous work we did this by relating EEG to the speech envelope and finding attention effects at a specific range of time lags from $195-230 \mathrm{~ms}$, suggesting that cocktail party attention operates at relatively long latencies, and, thus, beyond the earliest stages of acoustic processing (Power et al. 2012). Similarly, we sought here to explore how the attention effects we have observed on our phonetic features representation might vary across time lags. To do so, we first explored the possibility of examining the weights of a TRF that captures the unique variance in the EEG due to the phonetic features. To that end, we used cross-validation to fit a three-feature model based on $s, s D$, and $f o$ to best predict the EEG data. Then we subtracted this EEG prediction from the real EEG, and again used cross validation to model the residual EEG using only the phonetic feature representation. This model was able to predict significant variance in the residual EEG, and these 
predictions were affected by attention, supporting our earlier results. However, there was no clear effects of attention in the TRF weights themselves at any particular time lags. In fact, this was not terribly surprising. In our previous studies on cocktail party attention, we have found that attention effects on TRF weights themselves are generally much less robust and, thus, require a large amount of data (Power et al. 2012), relative to approaches based on EEG prediction or stimulus reconstruction using those TRFs (O'Sullivan et al. 2015).

With this in mind, we tried a second approach. Specifically, we repeated the approach of predicting EEG using phonetic features $f$, while controlling for $s, s D$, and $f o$. However, this time, rather than using a 0-300 ms interval of stimulus-response time lags, we ran the analysis separately for different, non-overlapping $50 \mathrm{~ms}$ windows from 0-300 ms. Figure 3C shows that attention has a significant effect on the phonetic feature-based predictions across a broad range of time lags, starting from around $50 \mathrm{~ms}$. However, the strongest effects of attention are visible from 150-250 ms, with weaker effects from 50-100, 100-150, and from 200-250 ms, and no attention effects at the shortest latencies of $0-50 \mathrm{~ms}$ (two-tailed Wilcoxon signed rank test; FDR corrected; 0-50 ms: $\mathrm{p}=0.1726, \mathrm{BF}_{10}=0.5468 ; 50-100 \mathrm{~ms}: \mathrm{p}=0.0166, \mathrm{BF}_{10}=3.8610 ; 100-150$ ms: $p=0.0017, B F_{10}=11.0430 ; 150-200 \mathrm{~ms}: \mathrm{p}=6.1035 \mathrm{e}-4, \mathrm{BF}_{10}=66.8325 ; 200-250 \mathrm{~ms}: \mathrm{p}=$ $\left.0.0031, \mathrm{BF}_{10}=32.4147 ; 250-300 \mathrm{~ms}: \mathrm{p}=0.0203, \mathrm{BF}_{10}=3.6121\right)$. This finding of strongest attention effects at time lags in the range $150-250 \mathrm{~ms}$ is largely consistent with earlier results that identified strong cocktail party attention effects at temporal latencies beyond $100 \mathrm{~ms}$ with limited effects at earlier latencies (Power et al. 2012; Puvvada and Simon 2017). One complicating factor here, however, is that exploring EEG predictions using narrow ranges of time delays is not necessarily as highly temporally resolved as it appears to be. This is because of the autocorrelational structure of the speech stimuli and of the EEG. The stimulus-data relationship at, say, $90 \mathrm{~ms}$, can be quite similar to that at $110 \mathrm{~ms}$. So the results need to be interpreted with some sensitivity to the likelihood of smearing in time.

[Fig 3 about here]

Figure 3 | (A) Unique predictive power for the acoustic and phonetic feature spaces under two attentional states. The features are as labelled on the horizontal axis. Statistical testing was carried out to identify attentional effects (two-tailed Wilcoxon signed rank; * $\mathrm{p}<0.05$, FDR corrected). (B) Topographic distribution of partial correlations, averaged across all subjects. 
428 (C) Unique predictive power for the phonetic features representation $(f)$ in $50 \mathrm{~ms}$ windows from

$4290-300 \mathrm{~ms}$, under two attentional states (two-tailed Wilcoxon signed rank test; ${ }^{*} \mathrm{p}<0.05$,

$430 * * \mathrm{p}<0.005$, FDR corrected).

431 Phonetic feature categories contribute predictive power beyond differentiating vowels and

432 consonants

433 Di Liberto et al., (2015) found a high degree of discriminability between EEG responses to 434 vowels and consonants. The phonetic feature representation used in the above analysis 435 information on the manner, place, and voicing of consonants, as well as the articulatory position 436 of vowels. We wondered how our results would be affected if - instead of using a 19437 dimensional vector - the phonemes were simply marked as vowels or consonants. We repeated 438 the partial correlation analysis described above and found that there was a significant decrease in 439 prediction accuracy when the information on specific articulatory features was left out in the case 440 of attended speech, but not for unattended speech (two-tailed Wilcoxon signed-rank test, $441 \mathrm{p}=0.011, \mathrm{z}=2.542$ ). Additionally, there was no longer a significant difference between the 442 prediction accuracies of attended and unattended stimuli (att_vc vs. unatt_vc; Wilcoxon signed443 rank test; $\mathrm{p}=0.4326 ; \mathrm{z}=0.7847$ )

[Fig 4 about here]

445

Figure 4 | A significant reduction in unique predictive power was observed for the attended 446 condition when the partial correlation analysis was repeated with phonemes only categorised as vowels or consonants (vc), as opposed to their underlying articulatory features (f) (two-tailed

448 Wilcoxon signed-rank test, ${ }^{*} \mathrm{p}=0.011, \mathrm{z}=2.542$ ). 


\section{Discussion}

There has been longstanding debate on how selective attention affects the processing of speech. Here, we set out to investigate attentional modulation at the pre-lexical level. In particular, based on earlier work (Di Liberto et al., 2015; Daube et al., 2019), we considered how two acoustic and two phonetic feature representations of attended and unattended speech were reflected in EEG responses to that speech. We found that, for attended speech, including a 19-dimensional phonetic features representation improved the prediction of the EEG responses beyond that obtained when only using acoustic features. This was not true for unattended speech. Furthermore, we found that the unique predictive power of the phonetic feature representation was enhanced for attended versus unattended speech. This was not true for any other feature. We contend that these findings make two important contributions to the literature. First, they contribute to the debate around pre-lexical speech processing in cortex. And second, they contribute to the longstanding debate on how selective attention affects the processing of speech.

In terms of prelexical speech processing, our study shows that a phonetic feature representation has unique predictive power when it comes to modelling responses to attended speech (Fig 2 \& Table 1). This suggests that processing attended, but not unattended speech, may involve a mapping from an acoustic to a categorical phonemic representation. This is a controversial idea. In particular, while linguistic theories and prominent speech processing models posit a mapping to phonetic features and/or phonemes as an intermediate stage between low-level acoustics and words (McClelland and Elman 1986; A. M. Liberman et al. 1967; Hickok and Poeppel 2007), this viewpoint is not unanimous. Some researchers question the necessity and existence of a mental representation at this level for comprehending speech meaning (Lotto and Holt, 2000). Indeed, there have been theories advocating for alternatives, including a different intermediate representational unit (e.g. syllables; Massaro 1974), or direct matching to the lexicon based on the comparison of acoustic representations (Goldinger 1998; Klatt 1989). In support of an acoustic-phonemic stage of processing, there has been evidence from behavioral and EEG studies that humans perceive phoneme categories. Liberman et al. (1957) found that discrimination within phoneme categorical boundaries is poorer than across them. Eimas et al. (1971) looked at infants' ability to discriminate syllables that differed in a voicing (phonetic) feature and found that infants also perceived categorical phonetic features. Additionally, 
Näätänen et al. (2001) found that the mismatch negativity (MMN), an event-related potential component that reflects discriminable change in some repetitive aspect of the ongoing auditory stimulation, is observed when the deviant stimulus is a phoneme prototype of a subject's native language relative to when it is a non-prototype. However, it has been argued that these studies used simple isolated units, and therefore their results could be due to the type of task and not reflective of everyday listening.

More recent neuroimaging studies have shown that a phonetic feature representation of speech can predict neural activity during listening to natural, continuous speech. Using high-density cortical surface electrodes, Mesgarani et al. (2014) found that the superior temporal gyrus (STG) selectively responds to phonetic features (their Figure 2-4). Di Liberto et al., (2015) and Khalighinejad et al., (2017) found evidence that non-invasive EEG activity reflects the categorization of speech into phonetic features. However, a recent paper challenged these findings and argued that neural responses to speech could be well explained by a model that is based entirely on acoustic features (Daube et al., 2019). Namely, they found that acoustic onsets made very similar predictions to the benchmark phonetic features, and that acoustic onsets explained parts of the neural response that phonetic features could not. Here, we show evidence to support the encoding of a rich array of phonetic features (certainly more than simply vowel and consonant categorization; Fig. 4) for attended speech. It is possible that we were able to find unique predictive power for these phonetic features because our study involved stimuli from two different talkers (a male and a female) while the stimuli used in Daube et al. (2019) were spoken by a single talker. As mentioned earlier, there is redundancy between speech acoustics and a phonetic feature representation because each phonetic feature has a characteristic spectrotemporal acoustic profile. This overlap can make it difficult to isolate the precise contribution of each predictor. Indeed, if there were no variation in spectro-temporal acoustics across each utterance of the same phoneme, the phonetic feature and spectrogram representations would be, effectively, identical. However, the more variation there is between utterances of the same phoneme (which will happen when including both male and female speakers), the greater the difference should be between the contribution of neurons that care about those variations (e.g., low-level "acoustic" neurons) and the contribution of neurons whose responses are invariant to the acoustic details and only dependent on phoneme category. As such, it may be that, when using stimuli with high intra-phoneme acoustic variation, including both acoustic and phonetic 
feature representations is of most benefit for predicting neural activity. Future work will investigate this idea further.

Interestingly, while our data support the idea of a mapping from an acoustic to a categorical phonemic representation during attended speech processing, we found no such evidence for unattended speech. Our Bayesian analysis $\left(\mathrm{BF}_{10}=0.4912>0.33\right.$, last row of Table 1) does not allow us to confidently assert that no such mapping takes place. For example, it could be that the generally lower prediction scores for unattended speech means that the signal-to-noise ratio of the data is just not high enough to detect subtle effects. That said, the EEG prediction scores for our individual (Fig 2B) and combined (Fig 2C) speech representations for unattended speech were all significantly above chance. Furthermore, the unique predictive power of the phonetic feature model for unattended speech was very low (Fig 3A, B). As such, it seems clear that, if it takes place at all, categorical processing of phonetic features for unattended speech is much reduced. Importantly, we contend that the lack of any evidence for phonetic feature processing of unattended speech actually further supports our claim that such phonetic feature processing is occurring for attended speech. In particular, if it were true that putative categorical phonemic responses to speech could be well explained by a model based entirely on acoustic features, then this would surely hold for both attended and unattended speech. That is, unless some acoustic features are more strongly modulated by attention than others. But we favor the interpretation that categorical linguistic processing (which surely happens) occurs for attended and not unattended speech and that this dichotomy extends from semantic (Broderick et al. 2018) and other linguistic levels (Brodbeck et al., 2018) down to the level of phoneme categorization.

As a final comment on this issue, we acknowledge that there are also other acoustic transformations of the auditory stimulus that have not been considered beyond the spectrogram and its derivative, as well as other theorized intermediate representations apart from phonemes/phonetic features that have not been tested. It is likely that some of these feature sets may overlap with our measure of phonetic features. As such, we cannot definitively prove the encoding of this measure in cortex. However, one reason the idea of mapping to words via an acoustic-phonetic stage has prevailed is due to its computational efficiency (Scharenborg et al. 2005). It is less obvious how the detection of acoustic onsets and other acoustic representations 
540 would lead to lexical access without an intermediate stage - and in particular, how such a model 541 would robustly generalize to new words and new speakers.

As to the cocktail party attention debate, our results suggest that attention differentially modulates cortical processing of acoustic and phonetic information. We found that the unique predictive power of phonetic features was modulated by attention, while the other feature spaces were not. This result is in line with a wealth of evidence for stronger attention effects at higher levels of the speech processing hierarchy. For example, there is no doubt that both attended and unattended speech will influence activity in the cochlea and the auditory nerve. But by the time one reaches auditory association areas like STG, only the attended speaker is represented (Mesgarani and Chang 2012; Zion Golumbic et al. 2013). Indeed, an important recent study (J. O'Sullivan et al. 2019) used simultaneous recordings in both primary auditory cortex (Heschl's gyrus; HG) and STG, to show that both attended and unattended speech are robustly represented in primary areas, with STG selectively representing the attended speaker. This lines up very well with our results. In particular, it is well established that spectro-temporal models of sounds can do a good job of explaining neural responses in primary auditory areas, but not in nonprimary auditory cortex (Norman-Haignere and McDermott 2018). Thus, we might expect our isolated acoustic speech processing indices to mostly reflect activity from primary auditory areas, which have been shown to be relatively unaffected by attention (J. O'Sullivan et al. 2019). Meanwhile STG is known to be selective for phonetic feature representations (Mesgarani et al. 2014) and activity in STG is strongly affected by attention (J. O'Sullivan et al. 2019). Thus, we might expect our isolated measures of phonetic feature processing to be strongly influenced by activity from STG, and thus, significantly affected by attention. This is the pattern of results that we have observed.

Additional support for our findings as evidence for a higher-level locus of attentional selection comes from research examining the latency of attention effects on EEG and MEG responses to speech. This approach has commonly focused on a rather general measure of speech processing that derives from indexing how the neural data track the speech envelope. Directly determining how much this speech processing is driven by acoustic versus speech-specific processing is not clear, although there is undoubtedly a very substantial acoustic contribution (Lalor et al. 2009; Howard and Poeppel 2010; Prinsloo and Lalor 2020). Nonetheless, researchers have examined 
components of these responses at different latencies as a proxy measure of processing at different hierarchical stages, and found that both attended and unattended speech are well represented in early components, with distinct responses to the attended speaker appearing only in late components (Ding and Simon 2012; Power et al. 2012; Puvvada and Simon 2017). Directly linking envelope tracking at different latencies with different representations of speech is a task for future research. Indeed, it may be quite an important task given the myriad efforts in recent years aimed at linking the effects of attention on behavior with those on a difficult-to-interpret neurophysiological measure that is likely substantially driven by attentionally-insensitive acoustic processing (e.g., Tune et al. 2020). Similarly, it may be that decoding of attentional selection from neural recordings will be improved by focusing on how those recordings reflect specific speech representations that are strongly affected by attention rather than the very general speech envelope (O’Sullivan et al. 2015).

Finally, it is important to add the caveat that our lack of an attention effect on isolated measures of acoustic processing is not evidence of their absence. Indeed, while the Bayes factor scores for the effect of attention on the unique predictive power of the $s$ and $s D$ models were both less than 1 , they were not less than 0.33 , meaning we cannot definitively assert that there are no attention effects at the acoustic level. It may well be that our analysis pipeline is not sensitive enough to detect small attention effects on those measures. Nonetheless, the fact that a clear difference was found for the phonetic feature representation supports the notion of stronger attention effects at higher levels of the speech processing hierarchy. The capacity of the brain to process information from multiple streams at any given moment in time is limited, so a longstanding question has concerned the extent to which unattended speech undergoes processing in cortex. Our results, considered together with studies focused on lexical (Brodbeck et al., 2018) and semantic (Broderick et al. 2018) processing, suggest it possible that attention is categorically selective for speaker-invariant representations and, at most, attenuates lower-level acoustic (speakerdependent) measures.

\section{References}

Ahissar, Ehud, Srikantan Nagarajan, Merav Ahissar, Athanassios Protopapas, Henry Mahncke, and Michael M. Merzenich. 2001. "Speech Comprehension Is Correlated with Temporal Response Patterns Recorded from Auditory Cortex." Proceedings of the National Academy of Sciences of the United States of America 98 (23): 13367-72. https://doi.org/10.1073/pnas.201400998. 
AJE, Kell, Yamins DLK, Shook EN, Norman-Haignere SV, and McDermott JH. 2018. “A TaskOptimized Neural Network Replicates Human Auditory Behavior, Predicts Brain Responses, and Reveals a Cortical Processing Hierarchy." Neuron 98 (3): 630-644.e16. https://doi.org/10.1016/J.NEURON.2018.03.044.

Algazi, V. R., Duda, R. O., Thompson, D. M., \& Avendano, C. (2001). The CIPIC HRTF database. Proceedings of the 2001 IEEE Workshop on the Applications of Signal Processing to Audio and Acoustics (Cat. No.01TH8575), 99-102.

Broadbent, D. (1958). Perception and Communication. Pergamon Press, London, UK

Brodbeck, Christian, L. Elliot Hong, and Jonathan Z. Simon. 2018. "Rapid Transformation from Auditory to Linguistic Representations of Continuous Speech." Current Biology 28 (24): 3976-3983.e5. https://doi.org/10.1016/j.cub.2018.10.042.

Broderick, Michael P., Andrew J. Anderson, Giovanni M. Di Liberto, Michael J. Crosse, and Edmund C. Lalor. 2018. "Electrophysiological Correlates of Semantic Dissimilarity Reflect the Comprehension of Natural, Narrative Speech." Current Biology 28 (5): 803-809.e3. https://doi.org/10.1016/j.cub.2018.01.080.

C, Tang, Hamilton LS, and Chang EF. 2017. "Intonational Speech Prosody Encoding in the Human Auditory Cortex.” Science (New York, N.Y.) 357 (6353): 797-801. https://doi.org/10.1126/SCIENCE.AAM8577.

Chang, Edward F, Jochem W Rieger, Keith Johnson, Mitchel S Berger, Nicholas M Barbaro, and Robert T Knight. 2010. "Categorical Speech Representation in Human Superior Temporal Gyrus.” Nature Neuroscience 13 (11): 1428-32. https://doi.org/10.1038/nn.2641.

Cherry, E Colin. 1953. "Some Experiments on the Recognition of Speech, with One and with Two Ears." Citation: The Journal of the Acoustical Society of America 25: 975. https://doi.org/10.1121/1.1907229.

Chomsky, N, and M Halle. 1968. "1968: The Sound Pattern of English. New York: Harper and Row."

Crosse, Michael J., Giovanni M. Di Liberto, Adam Bednar, and Edmund C. Lalor. 2016. "The Multivariate Temporal Response Function (MTRF) Toolbox: A MATLAB Toolbox for Relating Neural Signals to Continuous Stimuli." Frontiers in Human Neuroscience 10 (November): 604. https://doi.org/10.3389/fnhum.2016.00604.

Daube, Christoph, Robin A A Ince, and Joachim Gross. 2019. "Simple Acoustic Features Can Explain Phoneme-Based Predictions of Cortical Responses to Speech Article Simple Acoustic Features Can Explain Phoneme-Based Predictions of Cortical Responses to Speech.” Current Biology 29. https://doi.org/10.1016/j.cub.2019.04.067.

Davis, Matthew H., and Ingrid S. Johnsrude. 2003. "Hierarchical Processing in Spoken Language Comprehension." Journal of Neuroscience 23 (8): 3423-31. https://doi.org/10.1523/JNEUROSCI.23-08-03423.2003.

Delorme, Arnaud, and Scott Makeig. 2004. "EEGLAB: An Open Source Toolbox for Analysis of Single-Trial EEG Dynamics Including Independent Component Analysis." Journal of Neuroscience Methods 134 (1): 9-21. https://doi.org/10.1016/j.jneumeth.2003.10.009. 
Deutsch, J. A., and D. Deutsch. 1963. "Attention: Some Theoretical Considerations." Psychological Review 70 (1): 80-90. https://doi.org/10.1037/H0039515.

DeWitt, Iain, and Josef P. Rauschecker. 2012. "Phoneme and Word Recognition in the Auditory Ventral Stream." Proceedings of the National Academy of Sciences of the United States of America 109 (8): 2709. https://doi.org/10.1073/pnas.1113427109.

Di Liberto, Giovanni M., James A. O’Sullivan, and Edmund C. Lalor. 2015. "Low-Frequency Cortical Entrainment to Speech Reflects Phoneme-Level Processing." Current Biology 25 (19): 2457-65. https://doi.org/10.1016/J.CUB.2015.08.030.

Ding, Nai, and Jonathan Z. Simon. 2012. "Neural Coding of Continuous Speech in Auditory Cortex during Monaural and Dichotic Listening." Journal of Neurophysiology 107 (1): 7889. https://doi.org/10.1152/jn.00297.2011.

EC, Lalor, Power AJ, Reilly RB, and Foxe JJ. 2009. "Resolving Precise Temporal Processing Properties of the Auditory System Using Continuous Stimuli." Journal of Neurophysiology 102 (1): 349-59. https://doi.org/10.1152/JN.90896.2008.

ES, Teoh, and Lalor EC. 2019. "EEG Decoding of the Target Speaker in a Cocktail Party Scenario: Considerations Regarding Dynamic Switching of Talker Location." Journal of Neural Engineering 16 (3). https://doi.org/10.1088/1741-2552/AB0CF1.

ES, Teoh, Cappelloni MS, and Lalor EC. 2019. "Prosodic Pitch Processing Is Represented in Delta-Band EEG and Is Dissociable from the Cortical Tracking of Other Acoustic and Phonetic Features." The European Journal of Neuroscience 50 (11): 3831-42. https://doi.org/10.1111/EJN.14510.

Fisher, Ronald Aylmer, Sir, 1890-1962. 1924. "035: The Distribution of the Partial Correlation Coefficient.” https://digital.library.adelaide.edu.au/dspace/handle/2440/15182.

Gwilliams, Laura, Jean-Remi King, Alec Marantz, and David Poeppel. 2020. "Neural Dynamics of Phoneme Sequencing in Real Speech Jointly Encode Order and Invariant Content." BioRxiv, April, 2020.04.04.025684. https://doi.org/10.1101/2020.04.04.025684.

Hickok, Gregory, and David Poeppel. 2007. "The Cortical Organization of Speech Processing." Nature Reviews Neuroscience 8 (5): 393-402. https://doi.org/10.1038/nrn2113.

Holdgraf, Christopher R., Jochem W. Rieger, Cristiano Micheli, Stephanie Martin, Robert T. Knight, and Frederic E. Theunissen. 2017. "Encoding and Decoding Models in Cognitive Electrophysiology." Frontiers in Systems Neuroscience 11 (September): 61. https://doi.org/10.3389/FNSYS.2017.00061.

Johnston, William A., and Jolynn Wilson. 1980. "Perceptual Processing of Nontargets in an Attention Task." Memory \& Cognition 1980 8:4 8 (4): 372-77. https://doi.org/10.3758/BF03198277.

JR, Binder, Frost JA, Hammeke TA, Bellgowan PS, Springer JA, Kaufman JN, and Possing ET. 2000. "Human Temporal Lobe Activation by Speech and Nonspeech Sounds." Cerebral Cortex (New York, N.Y. : 1991) 10 (5): 512-28. https://doi.org/10.1093/CERCOR/10.5.512.

K, Okada, Rong F, Venezia J, Matchin W, Hsieh IH, Saberi K, Serences JT, and Hickok G. 
2010. "Hierarchical Organization of Human Auditory Cortex: Evidence from Acoustic Invariance in the Response to Intelligible Speech." Cerebral Cortex (New York, N.Y. : 1991) 20 (10): 2486-95. https://doi.org/10.1093/CERCOR/BHP318.

Khalighinejad, Bahar, Guilherme Cruzatto da Silva, and Nima Mesgarani. 2017. "Dynamic Encoding of Acoustic Features in Neural Responses to Continuous Speech." Journal of Neuroscience 37 (8): 2176-85. https://doi.org/10.1523/JNEUROSCI.2383-16.2017.

Lalor, Edmund C., and John J. Foxe. 2010. "Neural Responses to Uninterrupted Natural Speech Can Be Extracted with Precise Temporal Resolution." European Journal of Neuroscience 31 (1): 189-93. https://doi.org/10.1111/j.1460-9568.2009.07055.x.

Liberman, A. M., F. S. Cooper, D. P. Shankweiler, and M. Studdert-Kennedy. 1967. "Perception of the Speech Code." Psychological Review 74 (6): 431-61. https://doi.org/10.1037/H0020279.

Liberman, Alvin M., Katherine Safford Harris, Howard S. Hoffman, and Belver C. Griffith. 1957. "The Discrimination of Speech Sounds within and across Phoneme Boundaries." Journal of Experimental Psychology 54 (5): 358-68. https://doi.org/10.1037/H0044417.

Liberto, Giovanni M. Di, Daniel Wong, Gerda Ana Melnik, and Alain de Cheveigné. 2019. "Low-Frequency Cortical Responses to Natural Speech Reflect Probabilistic Phonotactics." NeuroImage 196 (August): 237-47. https://doi.org/10.1016/J.NEUROIMAGE.2019.04.037.

Lotto, A. J., \& Holt, L. (2000). The Illusion of the Phoneme. In: Billing, S.J., Boyle, J.P., \& Griffith, A.M. (eds), Chicago Linguistic Society, 35, 191-204.

Massaro, Dominic W. 1974. "Perceptual Units in Speech Recognition.” Journal of Experimental Psychology 102 (2): 199-208. https://doi.org/10.1037/H0035854.

McAuliffe, Michael, Michaela Socolof, Sarah Mihuc, Michael Wagner, and Morgan Sonderegger. 2017. "Montreal Forced Aligner: Trainable Text-Speech Alignment Using Kaldi." Proceedings of the Annual Conference of the International Speech Communication Association, INTERSPEECH 2017-August: 498-502. https://doi.org/10.21437/INTERSPEECH.2017-1386.

McClelland, James L., and Jeffrey L. Elman. 1986. "The TRACE Model of Speech Perception." Cognitive Psychology 18 (1): 1-86. https://doi.org/10.1016/0010-0285(86)90015-0.

Mesgarani, Nima, and Edward F. Chang. 2012. "Selective Cortical Representation of Attended Speaker in Multi-Talker Speech Perception." Nature. Nature Publishing Group. https://doi.org/10.1038/nature11020.

Mesgarani, Nima, Connie Cheung, Keith Johnson, and Edward F. Chang. 2014. "Phonetic Feature Encoding in Human Superior Temporal Gyrus." Science 343 (6174): 1006-10. https://doi.org/10.1126/science.1245994.

MF, Howard, and Poeppel D. 2010. "Discrimination of Speech Stimuli Based on Neuronal Response Phase Patterns Depends on Acoustics but Not Comprehension." Journal of Neurophysiology 104 (5): 2500-2511. https://doi.org/10.1152/JN.00251.2010.

Moray, Neville. 2018. "Attention in Dichotic Listening: Affective Cues and the Influence of 
Instructions:" Https://Doi.Org/10.1080/17470215908416289 11 (1): 56-60.

https://doi.org/10.1080/17470215908416289.

MR, Schädler, and Kollmeier B. 2015. "Separable Spectro-Temporal Gabor Filter Bank Features: Reducing the Complexity of Robust Features for Automatic Speech Recognition." The Journal of the Acoustical Society of America 137 (4): 2047-59. https://doi.org/10.1121/1.4916618.

Norman-Haignere, Sam V., and Josh H. McDermott. 2018. "Neural Responses to Natural and Model-Matched Stimuli Reveal Distinct Computations in Primary and Nonprimary Auditory Cortex." PLOS Biology 16 (12): e2005127. https://doi.org/10.1371/JOURNAL.PBIO.2005127.

O’Sullivan, James A., Alan J. Power, Nima Mesgarani, Siddharth Rajaram, John J. Foxe, Barbara G. Shinn-Cunningham, Malcolm Slaney, Shihab A. Shamma, and Edmund C. Lalor. 2015. "Attentional Selection in a Cocktail Party Environment Can Be Decoded from Single-Trial EEG." Cerebral Cortex 25 (7): 1697-1706. https://doi.org/10.1093/cercor/bht355.

O'Sullivan, James, Jose Herrero, Elliot Smith, Catherine Schevon, Guy M. McKhann, Sameer A Sheth, Ashesh D. Mehta, and Nima Mesgarani. 2019. "Hierarchical Encoding of Attended Auditory Objects in Multi-Talker Speech Perception." Neuron 104 (6): 1195-1209.e3. https://doi.org/10.1016/j.neuron.2019.09.007.

PD, Eimas, Siqueland ER, Jusczyk P, and Vigorito J. 1971. "Speech Perception in Infants." Science (New York, N.Y.) 171 (3968): 303-6. https://doi.org/10.1126/SCIENCE.171.3968.303.

Peelle, Jonathan E, Ingrid Johnsrude, and Matthew H Davis. 2010. "Hierarchical Processing for Speech in Human Auditory Cortex and Beyond." Frontiers in Human Neuroscience 4 (June): 51. https://doi.org/10.3389/FNHUM.2010.00051.

Power, Alan J., John J. Foxe, Emma-Jane Forde, Richard B. Reilly, and Edmund C. Lalor. 2012. "At What Time Is the Cocktail Party? A Late Locus of Selective Attention to Natural Speech." European Journal of Neuroscience 35 (9): 1497-1503. https://doi.org/10.1111/j.1460-9568.2012.08060.x.

Power, Alan J., Edmund C. Lalor, and Richard B. Reilly. 2011. "Endogenous Auditory Spatial Attention Modulates Obligatory Sensory Activity in Auditory Cortex." Cerebral Cortex 21 (6): 1223-30. https://doi.org/10.1093/CERCOR/BHQ233.

Prinsloo, Kevin D., and Edmund C. Lalor. 2020. "General Auditory and Speech-Specific Contributions to Cortical Envelope Tracking Revealed Using Auditory Chimeras." BioRxiv, October, 2020.10.21.348557. https://doi.org/10.1101/2020.10.21.348557.

Puvvada, Krishna C., and Jonathan Z. Simon. 2017. "Cortical Representations of Speech in a Multitalker Auditory Scene.” Journal of Neuroscience 37 (38): 9189-96. https://doi.org/10.1523/JNEUROSCI.0938-17.2017.

R, Näätänen. 2001. "The Perception of Speech Sounds by the Human Brain as Reflected by the Mismatch Negativity (MMN) and Its Magnetic Equivalent (MMNm)." Psychophysiology 
38 (1): 1-21. https://doi.org/10.1017/S0048577201000208.

Rauschecker, Josef P., and Sophie K. Scott. 2009. "Maps and Streams in the Auditory Cortex: Nonhuman Primates Illuminate Human Speech Processing.” Nature Neuroscience. https://doi.org/10.1038/nn.2331.

Reddy, Sravana, and James N. Stanford. 2015. “Toward Completely Automated Vowel Extraction: Introducing DARLA.” Linguistics Vanguard 1 (1): 15-28. https://doi.org/10.1515/LINGVAN-2015-0002.

Klatt, D. H. (1989). Review of selected models of speech perception. In Lexical representation and process (pp. 169-226). The MIT Press.

Richard Morey, Maintainer D. 2018. "Package 'BayesFactor' Title Computation of Bayes Factors for Common Designs."

Rosenfelder, Ingrid, Josef Fruehwald, Keelan Evanini, Scott Seyfarth, Kyle Gorman, Hilary Prichard, and Jiahong Yuan. 2014. "FAVE (Forced Alignment and Vowel Extraction) Suite Version 1.1.3," May. https://doi.org/10.5281/ZENODO.9846.

Rouder, Jeffrey N., Richard D. Morey, Paul L. Speckman, and Jordan M. Province. 2012. "Default Bayes Factors for ANOVA Designs." Journal of Mathematical Psychology 56 (5): 356-74. https://doi.org/10.1016/J.JMP.2012.08.001.

Scharenborg, Odette, Dennis Norris, Louis ten Bosch, and James M. McQueen. 2005. "How Should a Speech Recognizer Work?" Cognitive Science 29 (6): 867-918. https://doi.org/10.1207/S15516709COG0000_37.

SD, Goldinger. 1998. "Echoes of Echoes? An Episodic Theory of Lexical Access." Psychological Review 105 (2): 251-79. https://doi.org/10.1037/0033-295X.105.2.251.

Treisman, A. M. 1964. "Verbal cues, language, and meaning in selective attention." The American Journal of Psychology 77: 206-19. https://doi.org/10.2307/1420127.

Tune, Sarah, Mohsen Alavash, Lorenz Fiedler, and Jonas Obleser. 2020. "Neural Attention Filters Do Not Predict Behavioral Success in a Large Cohort of Aging Listeners." BioRxiv, May, 2020.05.20.105874. https://doi.org/10.1101/2020.05.20.105874.

Wagenmakers, Eric Jan, Ruud Wetzels, Denny Borsboom, and Han L.J. van der Maas. 2011. "Why Psychologists Must Change the Way They Analyze Their Data: The Case of Psi: Comment on Bem (2011)." Journal of Personality and Social Psychology 100 (3): 426-32. https://doi.org/10.1037/A0022790.

Y. Benjamini, Y. Hochberg 1995. "Controlling the False Discovery Rate : A Practical and Powerful Approach to Multiple Testing”. J. R. Stat. Soc. Series B Stat. Methodol., 57 (1995), pp. 289-300

Zion Golumbic, Elana M., Nai Ding, Stephan Bickel, Peter Lakatos, Catherine A. Schevon, Guy M. McKhann, Robert R. Goodman, et al. 2013. "Mechanisms Underlying Selective Neuronal Tracking of Attended Speech at a 'Cocktail Party."' Neuron 77 (5): 980-91. https://doi.org/10.1016/j.neuron.2012.12.037. 


\section{Acknowledgments}

801 This work was supported by a Career Development Award from Science Foundation Ireland 802 (15/CDA/3316), an Irish Research Council Government of Ireland Postgraduate Scholarship 803 (GOIPG/2015/3378), the Del Monte Neuroscience Institute at the University of Rochester and NIH grant 804 DC016297.

805 


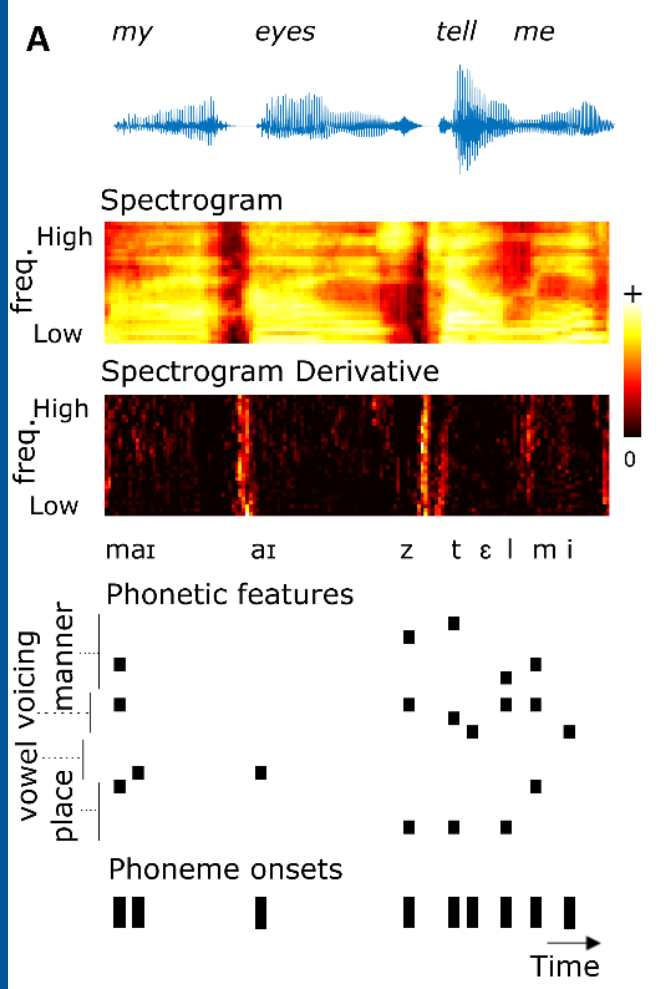

B

Talker 1 (Attended)

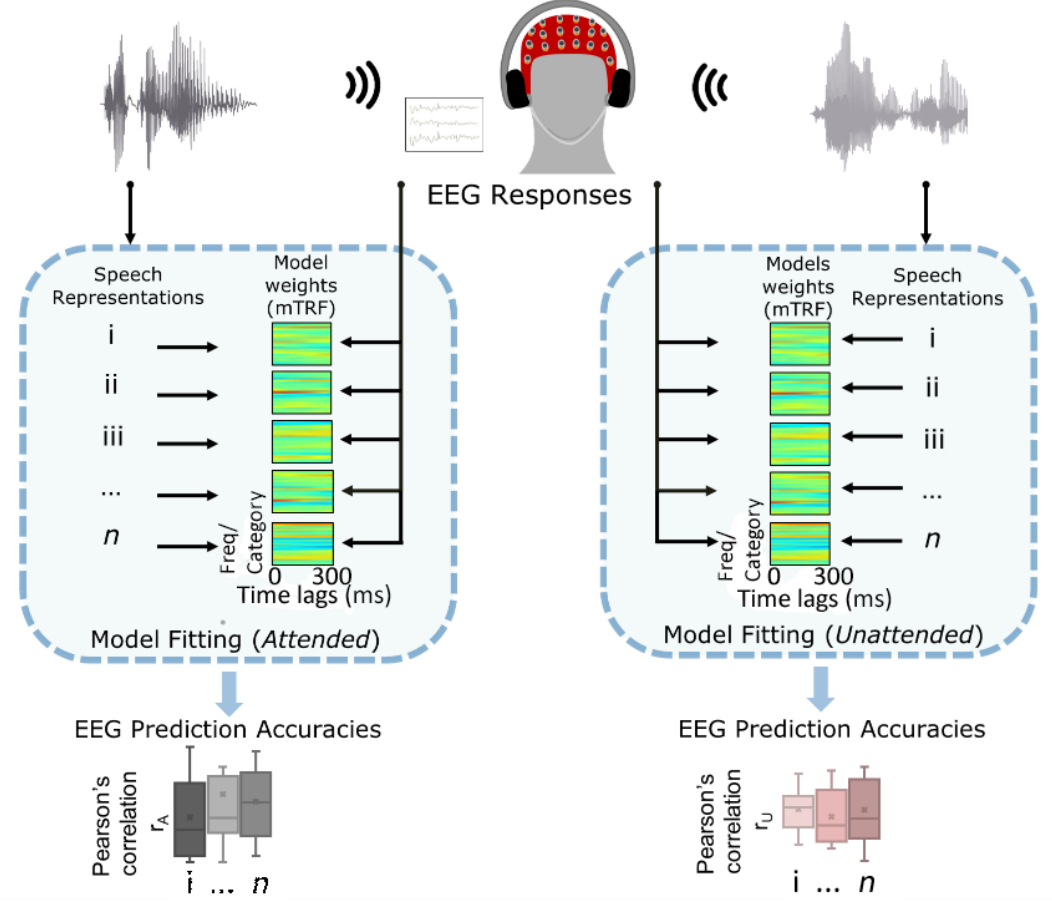


A Percentage of correct answers (\%) B
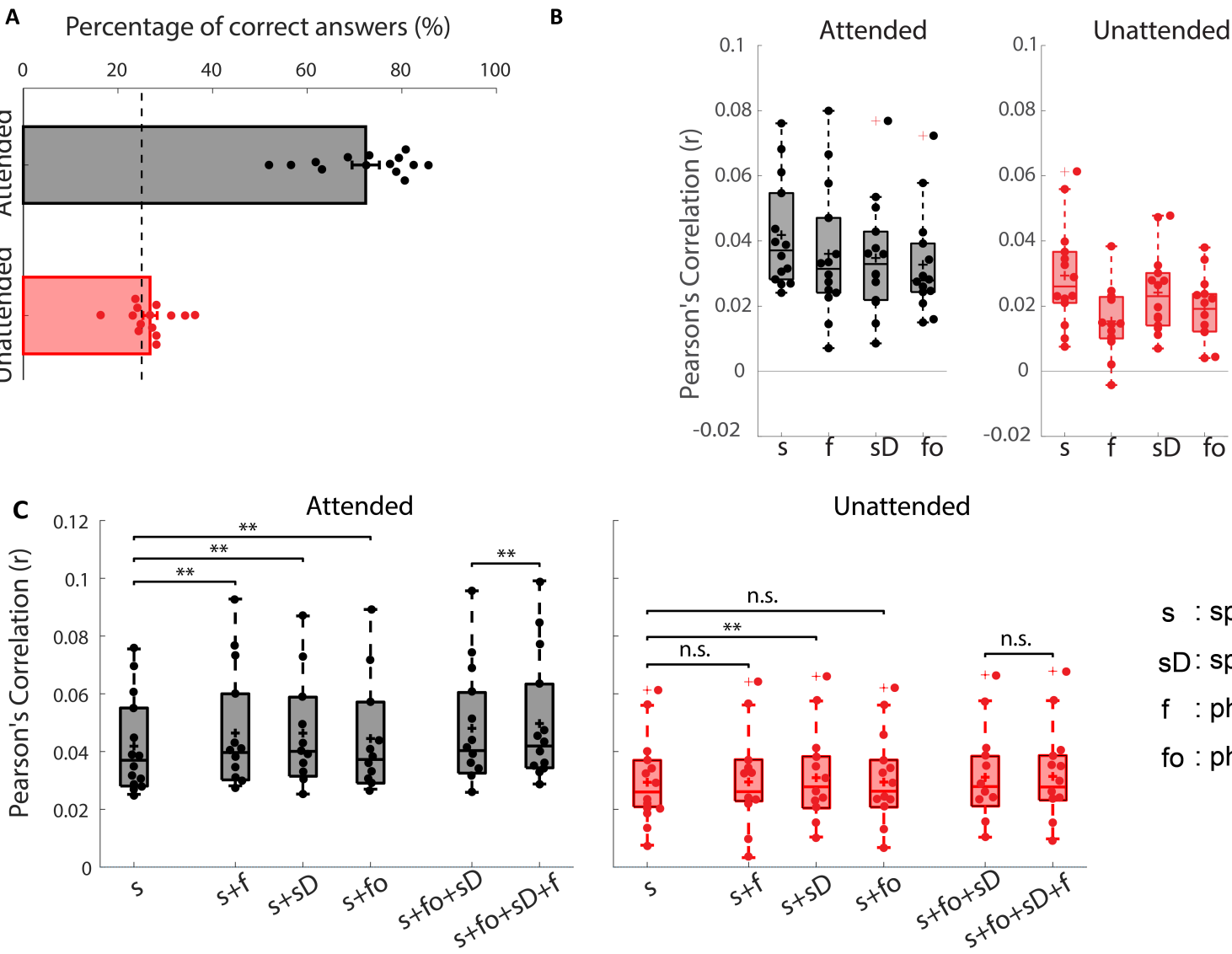

s : spectrogram

sD: spectrogram derivative

f : phonetic features

fo : phoneme onsets

0
0
0
2
2
2
2 


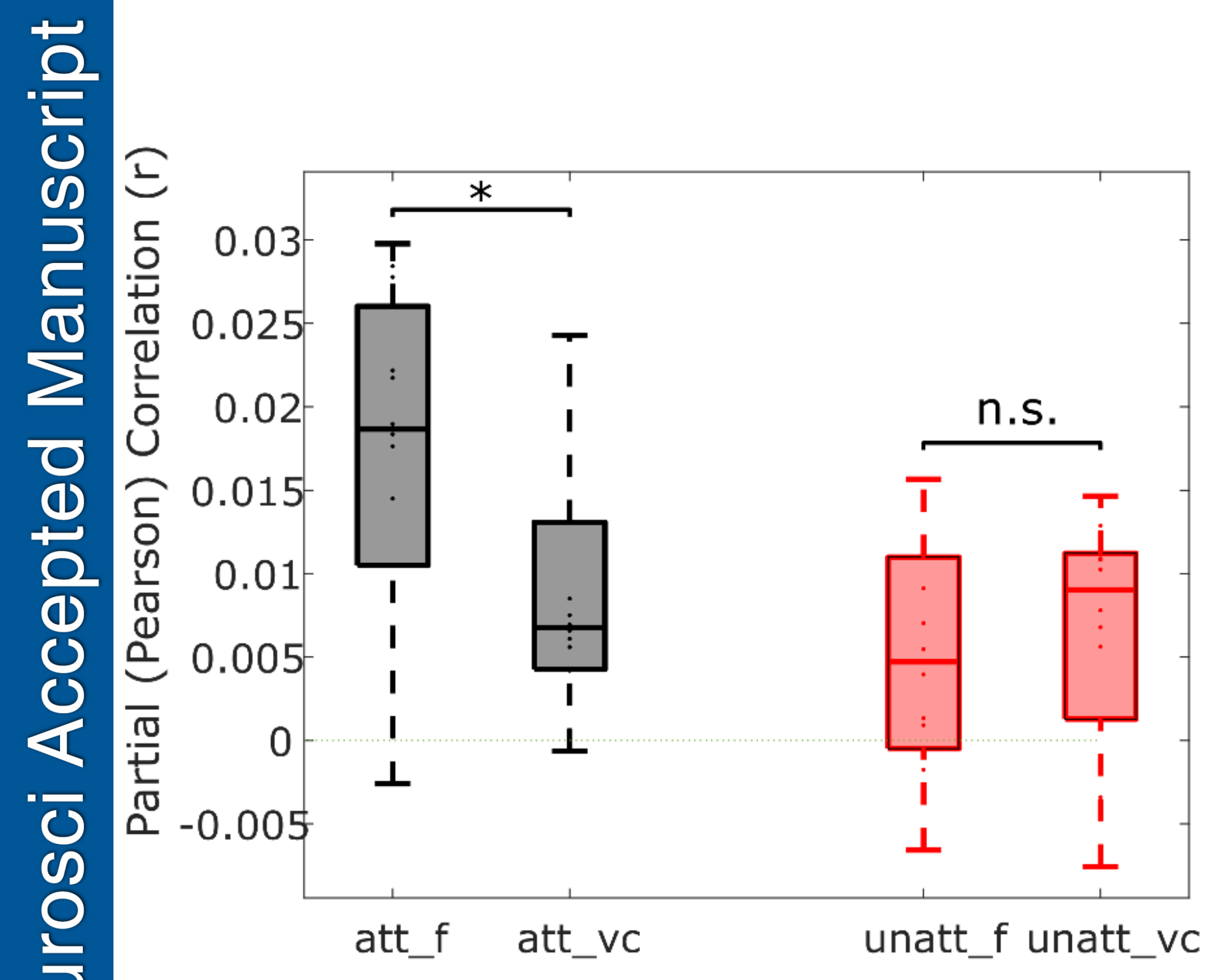

